\title{
Técnicas de interpolação geoespacial na estimativa do volume de Eucalyptus spp. na mesorregião sudeste paraense
}

O setor florestal brasileiro está em crescimento, consequentemente, aumenta a exigência sobre os silvicultores em relação à produtividade e gerenciamento da floresta, ocasionando a evolução do setor para a fronteira 4.0. Para tanto, há subsídio de ferramentas que proporcionem redução de custos e incremento da produtividade. Diante disso, objetivou-se com este trabalho avaliar o desempenho das análises geoestatísticas, sob diferentes semivariogramas, para modelar e mapear o volume de povoamentos de Eucalyptus spp. O estudo foi conduzido na Fazenda Piquiá, município de Paragominas, mesorregião Sudeste do Pará, onde foi realizado o inventário pré-corte em 35 talhões, com 694,40 ha. Foram alocadas e mensuradas 148 parcelas temporárias medindo $504 \mathrm{~m}^{2}$. A volumetria ( $\mathrm{m}^{3}$.ha1) de cada parcela do talhão foi interpolada por Krigagem Ordinária, com base nos semivariogramas esférico, exponencial, gaussiano e pentaesférico. 0 semivariograma Pentaesférico obteve o maior valor do alcance. O efeito pepita variou entre 0 e 537,56, para os semivariogramas Exponencial e Gaussiano, respectivamente. A dependência espacial foi forte para Esférico e Pentaesférico. Todos os semivariogramas apresentaram $R^{2}>0,90$. A RQME variou entre 30,77 e $31,43 \mathrm{~m}^{3}$.ha-1. O modelo geoespacial subestimou o volume total da área em $2,27 \%$, gerando um erro amostral de $1,68 \%$. O mapa geoestatístico denota que a distribuição dos volumes no povoamento não é homogênea, de tal modo que o uso de valores médios, não caracterizam corretamente a variabilidade da área. 0 modelo geoespacial krigagem ordinária com semivariograma Pentaesférico foi o que melhor se ajustou, servindo de ferramenta auxiliar aos inventários florestais tradicionais.

\section{Geospatial interpolation techniques in Eucalyptus spp. volume estimation in the southeast paraense's mesoregion}

\begin{abstract}
The Brazilian forest sector is growing, consequently, increasing the demand on foresters in relation to productivity and forest management, causing the sector's evolution to the 4.0 frontier. For this, there is subsidy of tools that provide cost reduction and productivity increase. The aim of this study was to evaluate the performance of geostatistical analyses, under different semivariograms, to model and map the volume of Eucalyptus spp. stands. The study was carried out at the Piquiá Farm, Paragominas municipality, southeast Pará mesoregion, where the pre-cut inventory was performed in 35 blocks, with 694.40 ha. A total of 148 temporary plots measuring $504 \mathrm{~m}^{2}$ were allocated and measured. The volumetry ( $\mathrm{m}^{3}$.ha-1) of each plot was interpolated by Ordinary Krigging, based on spherical, exponential, gaussian and pentaespherical semivariograms. The Pentaespherical semivariogram obtained the highest range value. The nugget effect ranged from 0 to 537.56 for Exponential and Gaussian semivariograms, respectively. Spatial dependence was strong for Spherical and Pentaespherical. All semivariograms had $\mathrm{R}^{2}>0.90$. The RMSE ranged from 30.77 to $31.43 \mathrm{~m}^{3}$.ha- 1 . The geospatial model underestimated the total volume by $2.27 \%$, generating a sampling error of $1.68 \%$. The geostatistical map shows that the distribution of volumes in the stand is not homogeneous, so that the mean values use does not correctly characterize the area variability. The ordinary krigging geospatial model with Pentaespherical semivariogram was the one that best fitted, serving as an auxiliary tool to traditional forest inventories.
\end{abstract}

Keywords: Krigging; Map; Semivariogram; Forestry 4.0.

Topic: Ciências Florestais

Reviewed anonymously in the process of blind peer.
Received: 02/08/2020

Approved: 17/09/2020

\author{
Marcio Roberto da Silva Melo iD \\ Universidade Federal Rural da Amazônia, Brasil \\ http://lattes.cnpq.br/2322429680520968 \\ http://orcid.org/0000-0002-2649-8869 \\ mrsmelo@yahoo.com.br \\ Gustavo Antonio Ruffeil Alves (D) \\ Universidade Federal Rural da Amazônia, Brasil \\ http://lattes.cnpq.br/1638279411609759 \\ http://orcid.org/0000-0003-2802-2036 \\ gustavo.ruffeil@ufra.edu.br
}

Referencing this:

CUNHA NETO, E. M.; ROCHA, J. E. C.; BEZERRA, J. C. F.; MELO, M. R. S.; ALVES, G. A. R.. Técnicas de interpolação geoespacial na estimativa do volume de Eucalyptus spp. na mesorregião sudeste paraense. Revista Ibero Americana de Ciências Ambientais, v.11, n.5, p.45-54, 2020. DOI: http://doi.org/10.6008/CBPC2179-6858.2020.005.0005 


\section{INTRODUÇÃO}

O setor florestal brasileiro registrou uma área de 7,83 milhões de hectares de reflorestamento, em 2018, participando de 6,9 \% do PIB Industrial nacional. Dentre as espécies utilizadas por este setor, destacamse as do gênero Eucalyptus, que ocupam 72,27 \% da área reflorestada do país (IBÁ, 2017). Inserido nesse contexto, o estado do Pará vem crescendo neste segmento, de tal forma que houve um incremento de 1,84 \% na área reflorestada do estado entre os anos de 2015 e 2017. No ano de 2018, foram identificados 208.074 ha de reflorestamentos no Pará, destes 72,62 \% cultivam as espécies do gênero Eucalyptus e 78,20 \% se localizam na mesorregião Sudeste do estado (IBGE, 2018).

Contudo, o crescimento do setor aumentou a exigência sobre os silvicultores em relação à produtividade e gerenciamento da atividade, de tal maneira que a silvicultura e o manejo florestal têm evoluído para a fronteira 4.0, com subsídio de ferramentas que proporcionem redução de custos e crescimento da produtividade, aliado à precisão de informações e gestão sustentável das florestas, bem como a precisão nas estimativas do estoque da floresta e suas características espaciais (CUNHA NETO et al., 2020; PELISSARI et al., 2017). Dentre essas ferramentas, destaca-se a geoestatística, por tornar viável a mensuração e identificação dos fatores limitantes para a produtividade dos povoamentos florestais, devido a predição de valores em locais não amostrados, bem como representação em mapas (PELISSARI et al., 2014a).

Dessa maneira, a geoestatística favorece o uso do sistema de informações geográficas (SIG), pois os mapas provenientes dessa técnica embasarão as decisões do silvicultor, fornecendo informações precisas dos atributos, possibilitando o uso sustentável dos insumos, evitando desperdício (SANTOS et al., 2017). Esta ferramenta se baseia na Teoria das Variáveis Regionalizadas (MATHERON, 1971), na qual a variável regionalizada é função numérica do fenômeno espacial, pelo qual a semivariância é a estatística básica que favorece a mensuração das relações estatísticas entre pontos distribuídos no espaço (PELISSARI et al., 2017).

Nas ciências florestais, diversos estudos comprovaram a eficiência da geoestatística, principalmente no que tange a estratificação de povoamentos (GUEDES et al., 2012), demarcação de zonas produtivas (CARVALHO et al., 2012), modelagem da altura (PELISSARI et al., 2014b), afilamento (PELISSARI et al., 2017), área basal (PELISSARIARI et al., 2017), biomassa (BARNI et al., 2016; BENÍTEZ et al., 2016) e volume (CUNHA NETO et al., 2019).

Dentre as técnicas geoestatísticas, a mais usual é a krigagem ordinária, por ser um algoritmo simples, aplicável para estimar um valor pontual em determinado local e interpolar para toda a área (YАMAMOTO et al., 2013). Além disso, com um semivariograma bem ajustado, a krigagem ordinária interpola dados em qualquer local da área com ausência de tendências (GREGO et al., 2014). Diante disso, objetivou-se com este trabalho avaliar o desempenho das análises geoestatísticas, sob diferentes semivariogramas, para modelar e mapear o volume de povoamentos de Eucalyptus spp. na Mesorregião sudeste do estado do Pará. 


\section{MATERIAIS E MÉTODOS}

\section{Caracterização de área}

O estudo foi conduzido na Fazenda Piquiá, localizada na às margens da Rodovia PA 125, km 42, no município de Paragominas, mesorregião Sudeste do Estado do Pará. Nesta fazenda foram selecionados 35 talhões, com 694,40 ha, nos quais estavam distribuídos três clones pertencentes ao gênero Eucalyptus, com sete anos de idade, sob o espaçamento $3 \mathrm{~m} \times 3 \mathrm{~m}$, tendo como coordenadas centrais o ponto $47^{\circ} 08^{\prime} 02,68^{\prime \prime}$ de latitude Sul e $03^{\circ} 16^{\prime} 01,54^{\prime \prime}$ de longitude Oeste (Figura 1). O clima é definido como "Aw", de acordo com a classificação de Köppen, com precipitação média anual de $1800 \mathrm{~mm}$. Ano-1 e temperatura média de 26 으 (ALVARES et al., 2013). O solo representativo da fazenda é o Latossolo Amarelo distrófico de textura muito arenosa (SANTOS et al., 2018).

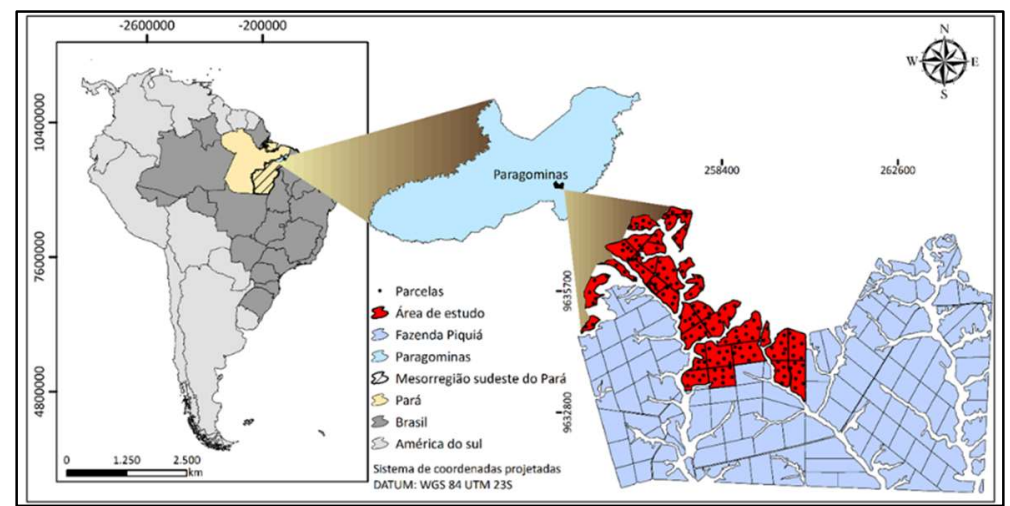

Figura 1: Área de estudo localizada na fazenda Piquiá, município de Paragominas, Mesorregião sudeste do estado do Pará.

\section{Banco de dados}

Nestes talhões foi realizado o inventário pré-corte, nos quais foram alocadas 148 parcelas temporárias medindo $21 \times 24 \mathrm{~m}\left(504 \mathrm{~m}^{2}\right)$. Estas parcelas foram alocadas com a intensidade de uma a cada cinco hectares. Nestas parcelas as árvores foram mensuradas quanto à circunferência a 1,30 m do solo (CAP) e a altura total, com auxílio da fita centimétrica e clinômetro digital Haglöf, respectivamente. Posteriormente, CAP foi transformado em DAP (diâmetro do fuste a 1,30 metros do solo) através da divisão entre CAP e a constante $\pi$. Adicionalmente, realizou-se a cubagem rigorosa das árvores, seguindo o método proposto por Smalian, com vistas no ajuste de equações volumétricas de regressão para corrigir os erros de inventário. Foram abatidas e cubadas quarenta e cinco árvores, sendo quinze indivíduos por clone, distribuídos em três classes diamétricas (Tabela 1).

Tabela 1: Distribuição diamétrica dos três clones instalados na fazenda Piquiá, Paragominas, Mesorregião sudeste do estado do Pará.

\begin{tabular}{|c|c|c|c|c|}
\hline \multicolumn{5}{|c|}{ Eucalyptus urophylla S.T Blake (C1) } \\
\hline Classe & $\mathrm{D}_{\mathrm{i}}(\mathrm{cm})$ & $\bar{X}(\mathrm{~cm})$ & $D_{s}(\mathrm{~cm})$ & $\mathrm{F}_{\mathrm{i}}$ \\
\hline 1 & 9,55 & 12,68 & 15,81 & \multirow{3}{*}{5} \\
\hline II & 15,81 & 18,94 & 22,07 & \\
\hline III & 22,07 & 25,20 & 28,33 & \\
\hline \multicolumn{5}{|c|}{ Eucalyptus urophylla S.T Blake (C2) } \\
\hline Classe & $\mathrm{Li}(\mathrm{cm})$ & $\bar{X}(\mathrm{~cm})$ & $\mathrm{Ls}(\mathrm{cm})$ & $\mathrm{F}_{\mathrm{i}}$ \\
\hline I & 9,68 & 12,68 & 15,68 & 5 \\
\hline
\end{tabular}




\begin{tabular}{|c|c|c|c|c|}
\hline II & 15,68 & 18,68 & 21,69 & \\
\hline III & 21,69 & 24,69 & 27,69 & \\
\hline \multicolumn{5}{|c|}{ Eucalyptus platyphylla F.Muell (C3) } \\
\hline Classe & $\mathrm{Li}(\mathrm{cm})$ & $\bar{X}(\mathrm{~cm})$ & $\operatorname{Ls}(\mathrm{cm})$ & $\mathrm{F}_{\mathrm{i}}$ \\
\hline 1 & 10,19 & 15,17 & 20,16 & \\
\hline II & 12,68 & 17,67 & 22,65 & 5 \\
\hline III & 15,17 & 20,16 & 25,15 & \\
\hline
\end{tabular}

Em que: $C=$ clone; $D_{i}=$ diâmetro mínimo; $\bar{X}=$ média dos diâmetros; $D_{s}=$ diâmetro máximo superior; $F_{i}=$ frequência de cada classe.

\section{Ajuste dos interpoladores geoestatísticos}

A volumetria $\left(\mathrm{m}^{3} \cdot \mathrm{ha}^{-1}\right)$ de cada parcela do talhão foi interpolada por Krigagem Ordinária, com base nos semivariogramas esférico, exponencial, gaussiano e pentaesférico. Foi realizado o teste de tendência anisotrópica, nas direções 0 ㅇ, 45, 90ㅇ e 135으, a fim de verificar se a direção possui influência no comportamento dos dados. As análises foram realizadas no software $R$ versão 3.61 (R CORE TEAM, 2019), com auxílio dos pacotes gstat (GRÄLER et al., 2016; PEBESMA, 2004) e geoR (RIBEIRO JUNIOR et al., 2020). Vale ressaltar que foram utilizados 12 pares de vizinhos para o cálculo dos semivariogramas.

Para a avaliação do ajuste utilizou-se as medidas estatísticas: coeficiente de determinação $\left(R^{2}\right)$, coeficiente de variação (CV) e grau de dependência espacial (GD). Esta variável foi analisada de acordo com a classificação de Cambardella et al. (1994) (Equação 1), pela qual são definidos com dependência espacial fraca os semivariogramas com GD $>75 \%$, moderada quando $25 \%<G D \leq 75 \%$ e forte quando o $G D \leq 25 \%$.

$$
G D=\frac{C_{0}}{C} \quad \text { (Equação 1) }
$$

\section{Validação dos interpoladores geoestatísticos}

Os quatro modelos geoestatísticos foram validados, utilizando a validação cruzada por Leave-oneout, o qual consiste em remover uma observação, ajustar o modelo com o restante dos dados e validá-lo em relação a observação removida do ajuste. Este processo é repetido para todas as observações.

A avaliação dos modelos validados foi realizada com as métricas estatísticas: Viés, Raiz quadrada do erro médio (RQME) e correlação linear de Pearson (r). Além disso, aplicou-se o teste $\mathrm{T}$, ao nível de $5 \%$ de significância, a fim de verificar se há evidencias estatísticas que comprovem a semelhança entre os volumes mensurados e estimados. Adicionalmente foi avaliada a dispersão dos resíduos entre os volumes, conforme a equação 2 .

$$
\text { Resíduo padronizado }=\frac{\left(V_{i}-\widehat{V}_{l}\right)}{\sqrt{s^{2}\left(\widehat{V}_{l}\right)}} \quad \text { (Equação 2) }
$$

Para selecionar o melhor modelo, atribuiu-se pesos as estatísticas utilizadas no ajuste e validação, de tal modo que o modelo com menor soma de pesos e melhor dispersão residual foi selecionado para representar o povoamento.

\section{RESULTADOS}

O inventário florestal pré-corte resultou em um baixo erro amostral relativo $(2,83 \%)$, com volume 
médio de 225,23 $\left(\mathrm{m}^{3} \cdot \mathrm{ha}^{-1}\right)$ e volume total de 153.558,24 $\mathrm{m}^{3}$. As parcelas apresentaram distribuição semelhantes à normal pelo teste de Shapiro-wilk ao nível de $5 \%$ de significância ( $w=0,989^{\text {ns }}$, valor $p=0,35$ ), com curtose de -0,183 (Figura 2). Vale ressaltar que a distância média entre as parcelas é 164,94 m e o coeficiente de variação entre seus volumes é $17,52 \%$.

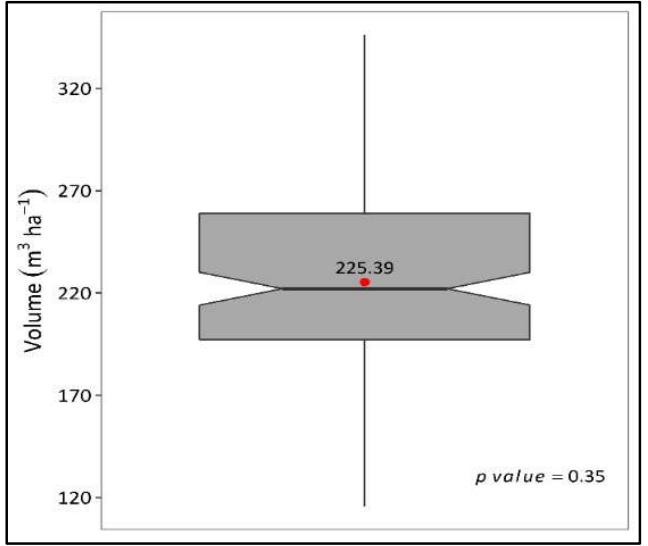

Figura 2: Boxplot do volume $\left(\mathrm{m}^{3} \cdot \mathrm{ha}^{-1}\right)$ mensurado na fazenda Piquiá, localizada em Paragominas, Mesorregião sudeste do estado do Pará.

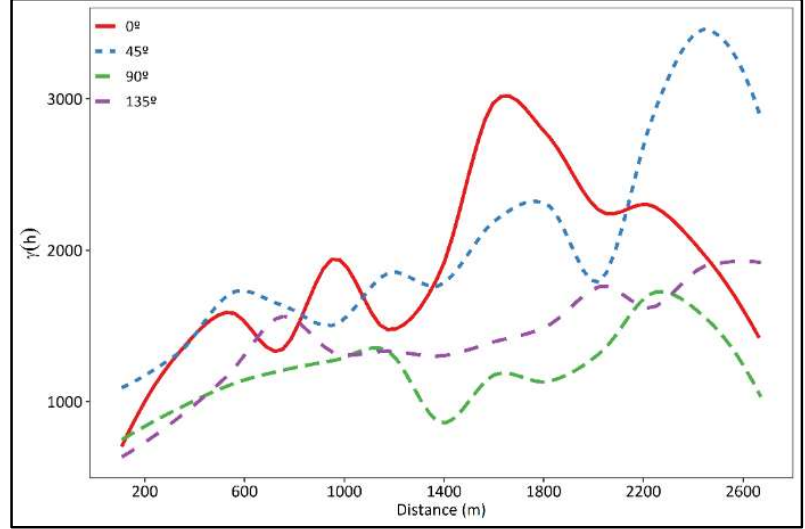

Figura 3: Teste do efeito anisotrópico nas parcelas mensuradas na fazenda Piquiá, localizada em Paragominas, Mesorregião sudeste do estado do Pará.

Os semivariogramas direcionais para 0으, 45 900 e 135 apresentaram um espalhamento das semivariancias em função da distância, porém as tendências são semelhantes, confirmando o comportamento isotrópico (Figura 3).

O semivariograma Pentaesférico obteve o maior valor do alcance, seguido do Esférico, enquanto o Exponencial apresentou menor valor deste parâmetro. O efeito pepita variou entre 0 e 537,56, para os semivariogramas Exponencial e Gaussiano, respectivamente. O maior patamar foi obtido para o Exponencial, enquanto Esférico e Pentaesférico apresentaram valores semelhantes dessa variável. Apesar disso, para o modelo Exponencial não foi verificada dependência espacial, enquanto Esférico e Pentaesférico obtiveram alta dependência espacial, já o Gaussiano apresentou dependência espacial moderada (Tabela 2).

Tabela 2: Resultados do ajuste e validação dos modelos geoestatísticos aplicados na estimativa do volume de madeira em pé de plantios de Eucalyptus spp. na mesorregião Sudeste do estado do Pará.

\begin{tabular}{|c|c|c|c|c|}
\hline \multirow{2}{*}{ Parâmetros } & \multicolumn{4}{|c|}{ Semivariograma } \\
\hline & Esférico & Exponencial & Gaussiano & Pentaesférico \\
\hline$a$ & 613,81 & 255,47 & 326,54 & 781,84 \\
\hline $\mathrm{C}_{0}$ & 259,89 & 0,00 & 537,56 & 278,44 \\
\hline C & 1221,67 & 1565,39 & 960,56 & 1221,69 \\
\hline GD (\%) & 21,27 & 0,00 & 55,96 & 22,79 \\
\hline$R^{2}$ & 0,969 & 0,977 & 0,970 & 0,971 \\
\hline CV (\%) & 11,36 & 11,79 & 11,21 & 11,33 \\
\hline Viés $\left(m^{3} \cdot h^{-1}\right)$ & 0,50 & 0,54 & 0,43 & 0,46 \\
\hline Viés (\%) & 0,22 & 0,24 & 0,19 & 0,20 \\
\hline RQME $\left(\mathrm{m}^{3} \cdot \mathrm{ha}^{-1}\right)$ & 31,43 & 30,77 & 31,33 & 31,05 \\
\hline RQME (\%) & 13,94 & 13,65 & 13,90 & 13,78 \\
\hline$r$ & 0,650 & 0,648 & 0,637 & 0,647 \\
\hline Teste-T & $0,85^{\text {ns }}$ & $0,83^{\text {ns }}$ & $0,87^{\mathrm{ns}}$ & $0,86^{\mathrm{ns}}$ \\
\hline Valor $p$ & $0,94^{\text {ns }}$ & $0,92^{\text {ns }}$ & $0,95^{\text {ns }}$ & $0,94^{\text {ns }}$ \\
\hline $\mathrm{P}$ & 16 & 16 & 15 & 13 \\
\hline
\end{tabular}

Em que: $a=$ alcance, $C_{0}=$ efeito pepita, $C=$ patamar, $R^{2}=$ coeficiente de determinação, $G D=$ grau de dependência espacial, $C V$ = coeficiente de variação, $\mathrm{RQME}=$ raiz quadrada do erro médio, $r=$ coeficiente de correlação de Pearson, $\mathrm{P}=$ soma dos pesos atribuídos as métricas estatísticas. 
Todos os semivariogramas apresentaram altos valores de $\mathrm{R}^{2}$ e coeficiente de variação inferiores a 12 \%. De maneira geral houve uma leve subestimativa entre os volumes, com viés variando entre 0,46 $\mathrm{m}^{3} . \mathrm{ha}^{-1} \mathrm{e}$ 0,54 $\mathrm{m}^{3}$.ha- ${ }^{1}$. A RQME variou entre $30,77 \mathrm{~m}^{3}$.ha- ${ }^{1}$ e $31,43 \mathrm{~m}^{3} \cdot$ ha- ${ }^{1}$, o que representa cerca de $13 \%$ de variação entre os volumes observados e preditos. Para todos os semivariogramas, a correlação entre os volumes foi moderada, no entanto, o teste $T$ evidenciou que os volumes estimados por geoestatística são estatisticamente semelhantes ao volume mensurado no inventário florestal (Tabela 2).

Entre os semivariogramas destacam-se o Gaussiano e Pentaesférico, por apresentarem menor soma de pesos, bem como dispersão reduzida dos valores observados em torno da linha média estimada, com ajustes satisfatórios para as estimativas espaciais do volume de eucalipto (Figura 4).

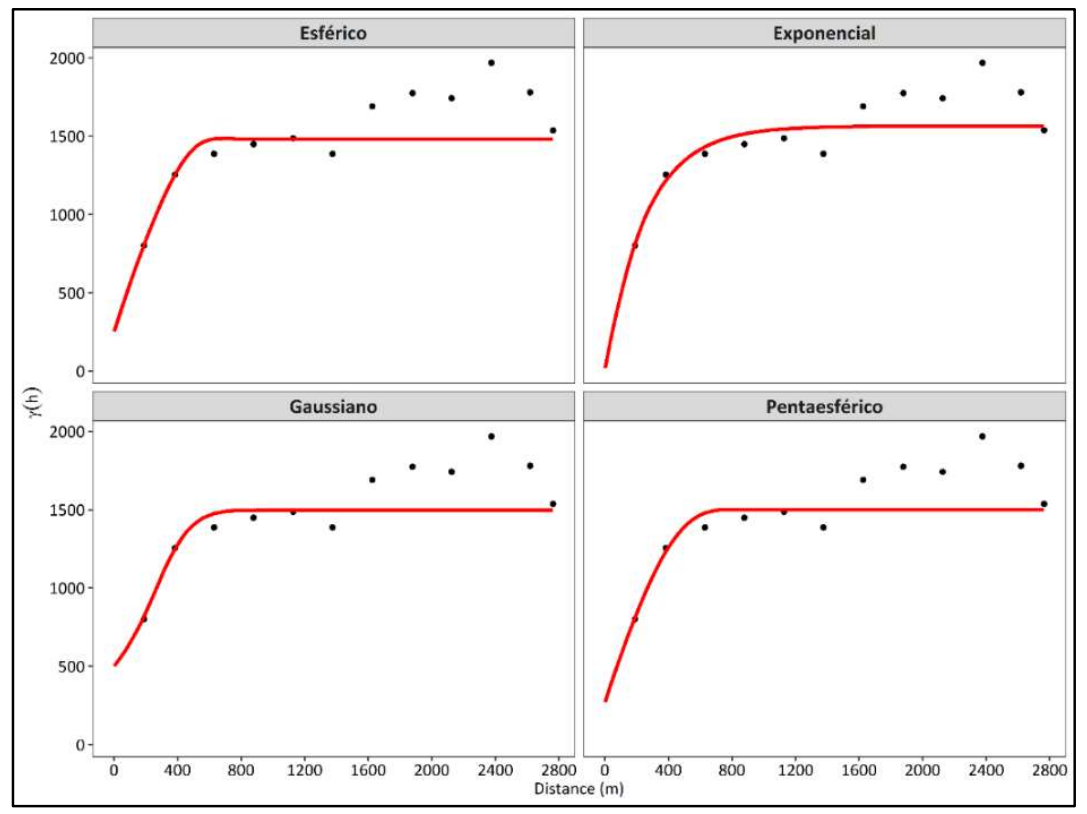

Figura 4: Semivariogramas testados na estimativa do volume de madeira em pé de plantios de Eucalyptus spp. na fazenda Piquiá, localizada em Paragominas, Mesorregião Sudeste do estado do Pará.

Os resíduos foram homocedásticos em todos os semivariogramas, mantendo-se entre -3 e 3 desvios. Não há presença de pontos influentes na dispersão residual (Figura 5a). A dispersão entre os volumes demonstrou comportamento característico de correlação moderada, com pontos dispersos entre a reta média dos volumes (Figura 5b).

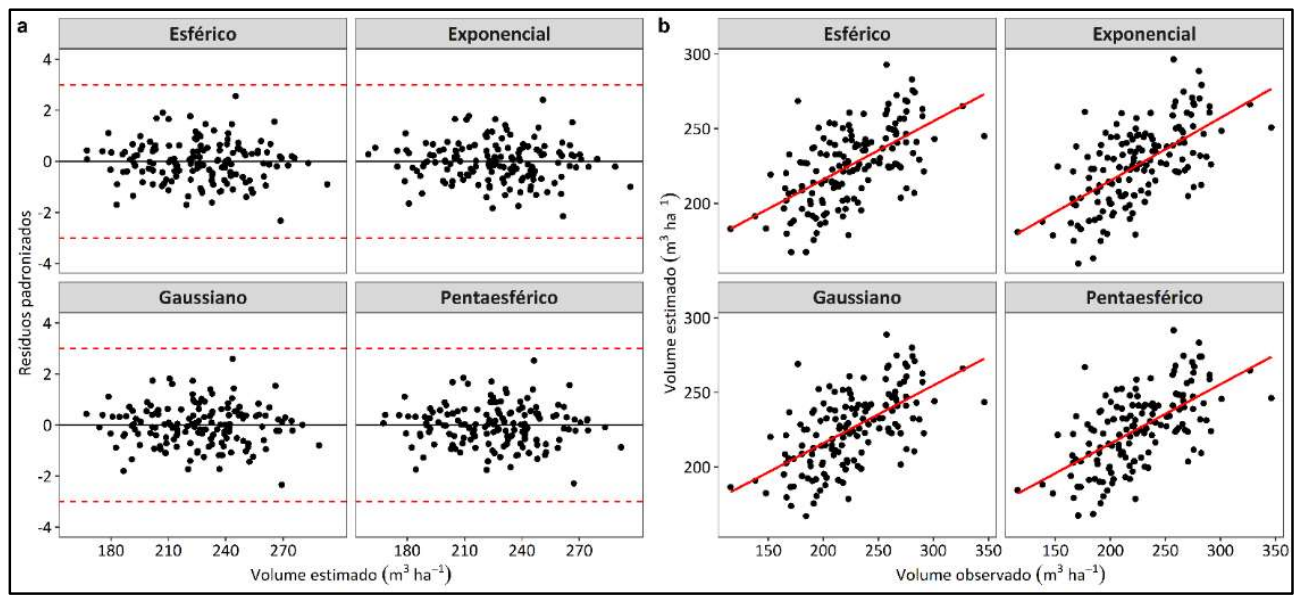

Figura 5: Dispersão do ajuste dos semivariogramas aplicados na estimativa do volume da madeira em pé de plantios de Eucalyptus spp. na fazenda Piquiá, localizada na Mesorregião sudeste do estado do Pará. 
O volume médio das parcelas por geoestatística foi de $225,66 \mathrm{~m}^{3}$, enquanto a projeção do volume total da área foi de $150.073,15 \mathrm{~m}^{3}$, uma diferença de 3.485,08 $\mathrm{m}^{3}$ em relação ao inventário florestal, ou seja, o modelo geoespacial subestimou o volume total da área em 2,27 \%, gerando um erro amostral de 1,68 \%. Vale ressaltar que apenas em oito talhões a dispersão residual foi superior a 0,40 desvios, com correlação de 0,93 entre o volume dos talhões, RQME de 18,99 \% e viés 2,42 \% (Figura 7).

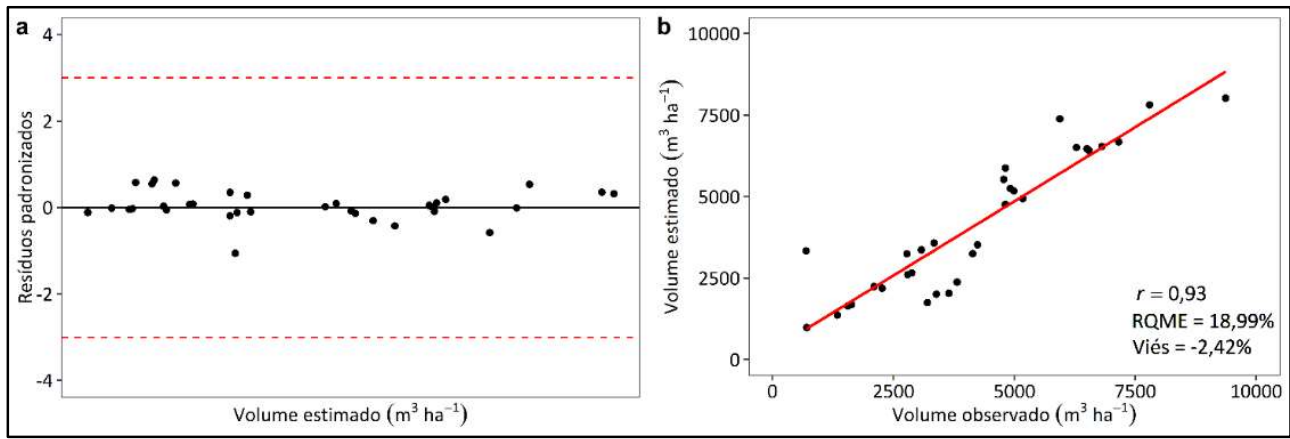

Figura 6: Dispersão dos erros e volumes para cada talhão da fazenda Piquiá, localizada em Paragominas, Mesorregião Sudeste do estado do Pará.

O mapa geoestatístico do semivariograma pentaesférico demonstrou a heterogeneidade do volume na área de estudo, pela qual as áreas de menor produtividade se localizam ao norte, enquanto as zonas mais produtivas estão na porção central, oeste e sudeste da fazenda (Figura 7).

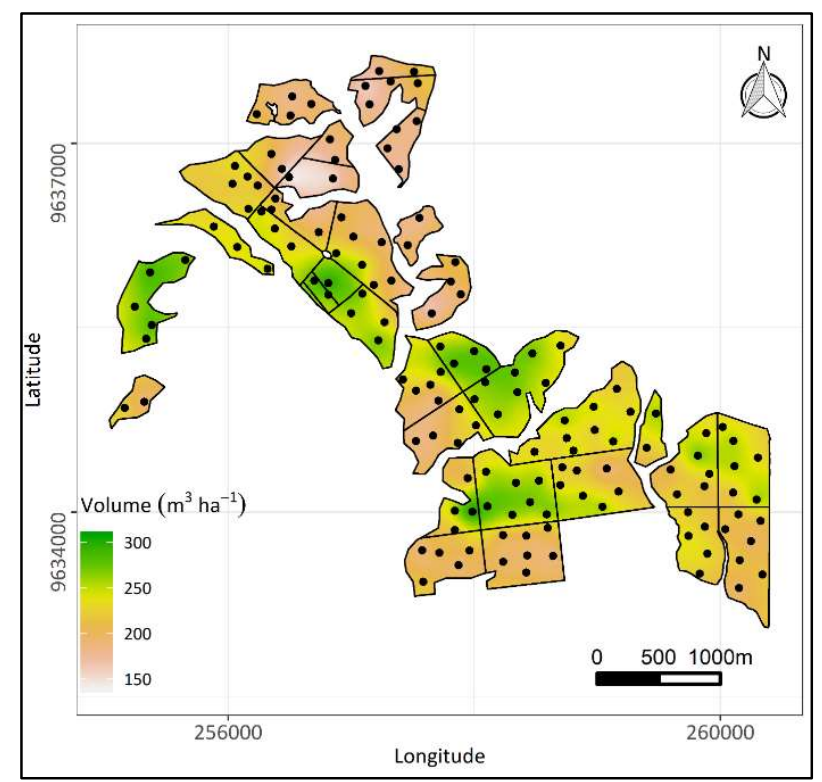

Figura 7: Mapa geoestatístico do volume de madeira em pé de plantios de Eucalyptus spp. estimados na fazenda Piquiá, localizada em Paragominas, Mesorregião Sudeste do estado do Pará.

\section{DISCUSSÃO}

O efeito pepita, ou variância aleatória é ocasionado por erros não identificados, de tal maneira que este demonstra a incerteza em distancias curtas, ou seja, quanto maior o efeito pepita, denota-se uma insuficiência de amostragem (YAMAMOTO et al., 2013), o que não ocorreu neste estudo. Além disso, o baixo valor desse parâmetro para os semivariogramas Esférico e Pentaesférico indicam que o ajuste destes foi satisfatório (PELISSARI et al., 2014b). Vale ressaltar que o $C_{0}$ destes semivariogramas (Tabela 2) apresentaram 
valores inferiores aos reportados por Carvalho et al. (2012) e Santos et al. (2017), o que ratifica a precisão do ajuste.

Adicionalmente, o alcance encontrado para o semivariograma Pentaesférico pode inferir alterações na amostragem utilizada neste estudo, uma vez que até a distância de 781,84 m há correlação espacial entre as parcelas. Assim, o alcance pode determinar a distância máxima entre parcelas, reduzindo a intensidade amostral do inventário (PELISSARI et al., 2014a). O elevado valor do alcance também implica na acurácia do ajuste por krigagem ordinária (MELLO et al., 2006), o que corrobora com os resultados deste estudo (Tabela 2).

O alto GD dos semivariogramas Esférico e Pentaesférico é confirmado pelo incremento e estabilização da semivariância (Figura 4), o que ressalta o ajuste adequado destes em relação à estimativa do volume no espaço (ROVEDA et al., 2016). O coeficiente de correlação, coeficiente de variação e a dispersão dos resíduos (Figura 5a) comprovam a precisão do ajuste destes semivariogramas, uma vez que o $R^{2}$ obteve valores superiores a 0,9 (CUNHA NETO et al., 2019; LUNDGREN et al., 2015; PELISSARIARI et al., 2017; SANTOS et al., 2017), os resíduos foram homocedasticos e o CV é classificado como baixo (GARCIA, 1989).

As estatísticas de validação Viés e RQME, apesar de apresentarem resultados satisfatórios, denotam as adversidades de adaptação dos modelos geoestatísticos aos padrões espaciais da variável interpolada (PELISSARIARI et al., 2017), o que provavelmente afetou a extrapolação do volume para a área total (Figura 6). Apesar disso, houve pouca diferença $(2,27 \%)$ entre o volume total estimado e o inventário florestal, bem como uma redução do erro de amostragem, corroborando com os resultados encontrados por Santos et al. (2017) e Cunha Neto et al. (2019), o que possibilita a redução dos custos, uma vez que se pode diminuir a intensidade amostral (MELLO et al., 2006).

O mapa geoestatístico (Figura 7) denota que a distribuição dos volumes no povoamento não é homogênea, de tal modo que o uso de valores médios, não caracterizam corretamente a variabilidade da área. Dessa forma, o conhecimento da variação do volume no espaço pode fomentar uma amostragem especifica para cada zona produtiva do povoamento, estratificando-o em subpopulações semelhantes, reduzindo a intensidade amostral, consequentemente os custos do inventário (GUEDES et al., 2012; PELISSARI et al., 2014a)

Este mesmo mapa pode favorecer o inventário pré-corte utilizando as parcelas permanentes do inventário florestal contínuo, uma vez que com a geoestatística, a variável é predita em locais não amostrados, determinando com precisão o volume da área (PELISSARI et al., 2017), o que é confirmando com os resultados obtidos neste estudo (Tabela 2, Figuras 5 e 6). Assim, a técnica de interpolação, além de fornecer dados imprescindíveis no que se refere a espacialização da produção do povoamento, possibilitou resultados pertinentes quanto o uso dessa ferramenta para inferências positivas e rápidas, de modo que o procedimento atende aos interesses do manejo florestal, proporcionando boas estimativas nas predições, assim como no auxílio do uso recursos/insumos para condução e reforma do povoamento, com base na silvicultura de precisão (LEAL et al., 2014). 


\section{CONCLUSÕES}

O modelo geoespacial krigagem ordinária com semivariograma Pentaesférico foi o que melhor se ajustou na estimativa do volume de madeira em pé de plantios de Eucalyptus spp. na fazenda Piquiá, mesorregião Sudeste do Estado do Pará, servindo de ferramenta auxiliar aos inventários florestais tradicionais, além de favorecer o planejamento específico das atividades silviculturais, maximizando a produção.

\section{REFERÊNCIAS}

ALVARES, C. A.; STAPE, J. L.; SENTELHAS, P. C.; GONÇALVES, J. L. M.; SPAROVEK, G.. Köppen's climate classification map for Brazil. Meteorologische Zeitschrift, v.22, n.6, p.711-728, 2013. DOI: http://doi.org/10.1127/0941-2948/2013/0507

BARNI, P. E.; MANZI, A. O.; CONDÉ, T. M.; BARBOSA, R. I.; FEARNSIDE, P. M.. Spatial distribution of forest biomass in Brazil's state of Roraima, northern Amazônia. Forest Ecology and Management, v.377, p.170181, 2016. DOI: http://doi.org/10.1016/i.foreco.2016.07.010

BENITTEZ, F. L.; ANDERSON, L. O.; FORMAGGIO, A. R.. Evaluation of geostatistical techniques to estimate the spatial distribution of aboveground biomass in the Amazon rainforest using high-resolution remote sensing data. Acta Amazônica, v.46, n.2, p.151-160, 2016. DOI: http://doi.org/10.1127/0941-2948/2013/0507

CAMBARDELLA, C. A. MOORMAN, T. B.; NOVAK, J. M.; PARKIN, T. B.; KARLEN, D. L.; TURCO, R. F.; KONOPKA, A. E.. Field-Scale Variability of Soil Properties in Central lowa Soils. Soil Science Society of America Journal, v.58, n.5, p.15011511, 1994.

CARVALHO, M. P. E.; MENDONÇA, V. Z.; PEREIRA, F. C. B. L.; ARF, M. V.; KAPPES, C.; DALCHIAVON, F. C.. Produtividade de madeira do eucalipto correlacionada com atributos do solo visando ao mapeamento de zonas específicas de manejo. Ciência Rural, v.42, n.10, p.1797-1803, 2012. DOI: http://doi.rog/10.1590/S0103-84782012005000078

CUNHA NETO, E. M.; NOGUEIRA JUNIOR, M. R.; MELO, M. R. S.; ROCHA, J. E. C.. Eucalyptus spp. volume determined through geospatial interpolation. Científica, v.47, n.4, p.434-40, 2019. DOI: http://doi.org/10.15361/19845529.2019v47n4p434-440

CUNHA NETO, E. M.; WALTER, L. S.; BERTI, A. L.; BRASIL, I. D. S.; MARTINS, V. C.; LINS, T. R. S.; SANTANA, G. M.; TERNES, G. B.; ARAUJO, E. C. G.; MOURA, M. M.; CORTE, A. P. D.; SANQUETTA, C. R.. Silvicultura 4.0. In: Avanços científicos e tecnológicos nas ciências agrárias. Ponta Grossa: Atena, 2020. p.148-156. DOI:

http://doi.org/10.22533/at.ed.61420190317

GARCIA, C. H.. Tabelas para classificação do coeficiente de variação. IPEF, Piracicaba, n.171, p.10, 1989.

GRÄLER, B.; PEBESMA, E.; HEUVELINK, G.. Spatio-Temporal Interpolation using gstat. The R Journal, v.8, n.1, p.204-218, 2016.
GREGO, C. R.; OLIVEIRA, R. P.; VIEIRA, S. R.. Geoestatística aplicada a agricultura de precisão Célia. Brasília: Embrapa, 2014. p.74-83.

GUEDES, I. C. L.; MELLO, J. M.; MELLO, C. R. OLIVEIRA, A. D.; SILVA, S. T.; SCOLFORRO, J. R. S.. Técnicas geoestatísticas e interpoladores espaciais na estratificação de povoamentos de Eucalyptus sp. Ciência Florestal, v.22, n.3, p.541-550, 2012. DOI: http://doi.org10.5902/198050986621

IBÁ. Indústria Brasileira de Árvores. Relatório 2017. IBÁ, 2017.

IBGE. Instituto Brasileiro de Geografia e Estatística. Produção da Extração Vegetal e da Silvicultura - PEVS. Rio de Janeiro: IBGE, 2018.

LEAL, F. A.; MATRICARDI, E. A. T.; MIGUEL, E. P.. Geostatistical interpolator to estimate volume in a settlement of Eucalyptus urophylla located in rio verde/goiás. Nucleus, v.11, n.1, p.153-159, 2014. DOI: http://doi.org/10.3738/1982.2278.1021

LUNDGREN, W. J. C.; SILVA, J. A. A.; FERREIRA, R. L. C.. Estimação de volume de madeira de eucalipto por cokrigagem, krigagem e regressão. Cerne, v.21, n.2, p.243250, 2015. DOI: http://doi.org/10.1590/01047760201521021532

MATHERON, G.. The theory of regionalized variables and its applications. Fontainebleau: École Nationale Supérieure des Mines de Paris, 1971.

MELLO, J. M.; OLIVEIRA, M. S.; BATISTA, J. L. F.; JUSTINIANO JÚNIOR, P. R.; KANEGAE JÚNIOR, H.. Uso Do Estimador Geoestatístico Para Predição Volumétrica Por Talhão. Floresta, v.36, n.2, p.251-260, 2006. DOI: http://doi.org/10.5380/rf.v36i2.6454

PEBESMA, E. J.. Multivariable geostatistics in S: the gstat package $\$$. Computers and geosciences, v.30, p.683-691, 2004.

PELISSARI, A. L.; FIGUEIREDO FILHO, A.; CALDEIRA, S. F.; MACHADO, S. A.. Geoestatística aplicada ao manejo de povoamentos florestais de teca, em períodos pré-desbaste seletivo, no estado do mato grosso. Rev. Bras. Biom., v.32, n.3, p.430-444, 2014a.

PELISSARI, A. L.; FIGUEIREDO FILHO, A.; CALDEIRA, S. F.; MACHADO, S. A.. Geostatistic Applied to Spatial Modeling of Hypsometric Relationships in Forest Stands. American 
International Journal of Contemporary Research, v.4, n.7, p.67-76, 2014b.

PELISSARI, A. L.; ROVEDA, M. CALDEIRA, S. F.; SANQUETTA, C. R.; CORTE, A. P. D.; RODRIGUES, C. K.. Stem taper modeling and timber volume estimation. Cerne, v.23, n.1, p.115-122, 2017. DOI:

http://doi.org/10.1590/01047760201723012291

PELISSARIARI, A. L.; FIGUEIREDO FILHO, A. PÉLLICO NETTO, S.; EBLING, A. A.; ROVEDA, M.; SANQUETTA, C. R. Geostatistical modeling applied to spatiotemporal dynamics of successional tree species groups in a natural Mixed Tropical Forest. Ecological Indicators, v.78, p.1-7, 2017. DOI: http://doi.org/10.1016/j.ecolind.2017.02.044

R CORE TEAM. R: A language and environment for statistical computing. Vienna: R Foundation for Statistical Computing, 2019.

RIBEIRO JUNIOR, P. J.; DIGGLE, P. J.; SCHLATHER, M.; BIVAND, R.; RIPLEY, B.. GeoR: Analysis of Geostatistical Data. $\mathrm{R}$ package, 2020
ROVEDA, M.; FIGUEIREDO FILHO, A.; PELISSARI, A. L.; SANTOS, R. M. M.. Spatial continuity in a Mixed Ombrophilous Forest with different size and shape of sample units. Cerne, v.22, n.2, p.189-196, 2016. DOI: http://doi.org/10.1590/01047760201622022139

SANTOS, M. C.; ROVEDA, M.; ZANON, M. L. B.; FIGUEIREDO FILHO, A.; ROIK, M.; PACHECO, J. M.; SCAVINSKI, V.. Inventário florestal utilizando técnicas de silvicultura de precisão em povoamentos de Eucalyptus grandis hill ex maiden. Floresta e Ambiente, v.24, 2017. DOI: http://doi.org/10.1590/2179-8087.082714

SANTOS, H. G.; JACOMINE, P. K. T.; ANJOS, L. H. C.; OLIVEIRA, V. A.; LUMBRERAS, J. F.; COELHO, M. R.; ALMEIDA, J. A.; ARAÚJO FILHO, J. C.; OLIVEIRA, J. B.; CUNHA, T. J. F.. Sistema brasileiro de classificação de solos. 5 ed. Brasília: Embrapa, 2018.

YAMAMOTO, J. K.; LANDIM, P. M. B.. Geoestatística: conceitos e aplicações. São Paulo: Oficina de textos, 2013.

A CBPC - Companhia Brasileira de Produção Científica (CNPJ: 11.221.422/0001-03) detém os direitos materiais desta publicação. Os direitos referem-se à publicação do trabalho em qualquer parte do mundo, incluindo os direitos às renovaç̃oses, expansões e disseminações da contribuiç̃o, bem como outros direitos subsidiários. Todos os trabalhos publicados eletronicamente poderão posteriormente ser publicados em coletâneas impressas sob coordenação da Sustenere Publishing, da Companhia Brasileira de Produção Científica e seus parceiros autorizados. Os (as) autores (as) preservam os direitos autorais, mas não têm permissão para a publicação da contribuição em outro meio, impresso ou digital, em português ou em tradução. 